\title{
Broadband Frequency Domain-Based Air Interfaces for 4G Cellular Wireless Systems
}

\author{
Inderjeet Kaur \\ Dept of CSE \\ Ajay Kumar Garg Engineering College, \\ Ghaziabad, India.
}

\begin{abstract}
Future-generation cellular wireless systems, transporting high bit rates in non-ideal radio propagation environments, must be robust to severe frequency selective multipath. Further requirements include moderate terminal and base station hardware costs, high spectral efficiency, and scalability of the cost of terminals with respect to their maximum bit rate capabilities. Reconfigurable air interfaces, based on frequency domain transmission and reception methods, best meet these requirements, by adaptively selecting the uplink and downlink modulation and multiple access scheme that is most appropriate for the channel, interference, traffic and cost constraints. This approach also leads to a general unified framework for possible air interfaces.
\end{abstract}

\section{Introduction-Requirements}

Future-generation wireless access systems will be mostly IP packet-based and must provide satisfactory quality of service with respect to: bit and packet error rate, end to end and link delays, outage probability, packet loss and packet delay variation for different traffic classes.

Multimedia services with peak user bit rates ranging from a few $\mathrm{Kb} / \mathrm{s}$ up to at least several tens of $\mathrm{Mb} / \mathrm{s}$ are foreseen. Aggregate data rates will be on the order of 50 to $100 \mathrm{Mb} / \mathrm{s}$, or even higher in hot spots [MLM02], [KB02], [ITU03]. Transmission will occur in varied radio propagation environments, with moving terminals, and with sharing of the radio spectrum by many user terminals. Future systems must use spectrum-efficient air interface technologies which can compensate for the severe frequency selectivity and other radio impairments, while keeping signal processing complexity within reasonable limits.

Spectrum availability for next generation broadband wireless services is presently uncertain. All wireless user terminals should be as efficient as possible in spectrum utilization. Efficient spectrum utilization may require cognitive radio operation, in which the transmitted signal bandwidth and center frequency or frequencies must be adaptively and dynamically adjusted in response to the current interference environment, shared by many user terminals and perhaps multiple service providers. Intelligent spectrum-sharing capability and contextawareness requirements will affect the air interface design, as well as dynamic resource allocation, scheduling and medium access control (MAC) design. Reconfigurability in response to varying network and user requirements will be made possible by software defined radio technologies.
An important additional requirement is low cost: low terminal cost and power consumption for subscribers, high spectral efficiency and coverage, and ease of deployment for service providers. Concentrating as much signal processing as possible in the base station or access point, instead of the user terminal, is a step in this direction. The choice of modulation and coding schemes can significantly influence performance and cost for a given range of bit rates.

Terminal specifications should be scalable with respect to user bandwidth requirements. Simple user terminals may be limited to low bit rates, while more sophisticated user terminals will be able to employ the full range of bit rates on demand. The cost and complexity of terminals restricted to low bit rates should not be driven by the requirements and system architecture of high bit rate terminals. Systems should also be flexible with respect to duplex traffic symmetry or asymmetry.

In this White Paper, we outline a generalized multicarrier approach to link transmission, reception and multiple access for future-generation systems in Sections 2 and 3. This class of signals includes parallel modulation (such as OFDM, OFDMA, MC-CDMA, SS-MC-MA) and serial modulation (such as conventional single carrier, single carrier DS-CDMA, FDOSS). Common signal processing operations within this general class facilitate reconfigurability of the air interface in response to user requirements and the radio environment. Section 4 discusses bit error rate performance characteristics of parallel and serial modulated signals. Section 5 discusses spread spectrum (CDMA) signalling within these two main classes. In Sections 6 and 7 , we consider the impairments of phase noise, frequency offset and power amplifier nonlinearities and their effects on these types of signals. Spectrum flexibility, including transmission over several disjoint sub-bands in a crowded spectrum, is readily achieved with frequency domain signalling, and is discussed in Section 8. Sections 9 and 10 complete the White Paper with brief discussions of further research issues and a summary, respectively.

\section{Frequency Domain- Based Systems}

In $100 \mathrm{Mb} / \mathrm{s}$ systems, with delay spreads of several $\mu \mathrm{s}$, the application of traditional time domain equalization methods would require prohibitively complex and high speed signal processing, since the intersymbol interference (ISI) could span hundreds of data bits. A better equalization performance/complexity tradeoff is obtained by doing transmission and reception operations on a block by block basis in the frequency domain, using discrete Fourier transform (DFT) 
processing, for which the signal processing complexity per bit increases only logarithmically with ISI span, when the DFT is implemented as a fast Fourier transform (FFT). Modulation and equalization schemes in which signal processing is done in the frequency domain include "parallel" schemes such as orthogonal frequency division multiplexing (OFDM) [CS00], [VP00] and its variants [FK03], and "serial modulation" schemes such as single carrier with frequency domain equalization (SC-FDE) [SKJ94], [FAB+02].

\subsection{Parallel Modulation Methods}

In "parallel" modulation methods, coded data symbols are transmitted in parallel on narrowband subcarriers. Prime examples include OFDM, OFDMA (orthogonal frequency division multiple access [SLK97], [VP00], [KR02]), MCCDMA (multicarrier code division multiple access [FK03], and SS-MC-MA (spread spectrum multicarrier multiple access) [Kai98], [Kai02]), The transmitter's modulation process is carried out on blocks of data symbols (typically QAM quadrature amplitude modulation), using a computationallyefficient inverse FFT (IFFT) operation. At the receiver a FFT operation decomposes each received signal block into the narrowband subcarrier components, which are independently equalized and passed on to the decoder. A cyclic prefix (CP) precedes each transmitted block to act as a guard interval to avoid inter-block ISI, and to produce the appearance of cyclic convolution by the channel impulse response, thus facilitating the FFT operation. The $\mathrm{CP}$ is a copy of the end of the transmitted block, and its length should be at least equal to the maximum expected channel delay spread.

The CP (and maximum expected delay spread) should be a small fraction (e.g. <1/8) of the FFT block length for two main reasons: (1) to minimize the percentage of overhead caused by the CP; (2) to ensure that the inter-subcarrier frequency spacing is much less than the channel's coherence bandwidth; then each transmitted subcarrier passes through an essentially nonfrequency selective channel, and therefore inter-subcarrier interference is minimized.

Since time domain cyclic convolution is equivalent to frequency domain multiplication of FFTs, equalization is carried out by a simple complex gain multiplication on each received subcarrier. It can be incorporated within the decoding process in coded OFDM. Coding and inter- or intra-block interleaving are essential for OFDM systems used on frequency-selective channels; the decoder sees the equivalent of a fast-fading channel, since each coded data symbol emerging from the equalizer has a different instantaneous signal to noise ratio (SNR), depending on the channel gain at its subcarrier frequency. Channel state information about the gain of each OFDM subcarrier is generally used in decoding, since it significantly enhances bit error rate performance. The coding/decoding process provides frequency diversity, which is essential for OFDM or OFDMA systems used on frequency selective channels.

Sequences of zeroes of the same length as the $\mathrm{CP}$ can replace the CP's separating successive blocks. This approach, called zeropadding $[\mathrm{MDD}+00]$ requires slightly more complex equalization, but has the advantage that nulls in the channel's frequency response do not hinder equalization. A further variation, called pseudo-random postfix [MCD+03], [MDC+04], [MCD04], [MCM+05] replaces each zero sequence with a fixed sequence of training symbols multiplied by a pseudo-random rotation factor to avoid spectral peaks. The interspersed training sequences combine the functions of periodic channel estimation and prevention of inter-block interference. This combination reduces overhead. Equalization is similar to methods employed for zero-padding.

The CP overhead is eliminated completely in a scheme called OFDM/IOTA, [FP93], [LJG02]. In it the data symbols are transmitted using offset QAM modulation, with filtering by a Gaussian-like waveform following the inverse FFT operation.

\subsection{Serial Modulation Methods}

Equalization in the frequency domain, using computationallyefficient FFT and inverse FFT operations, can also be applied to traditional serial modulation, or "single carrier" modulation schemes, in which data symbols are transmitted sequentially, with a high symbol rate, on a single carrier. In serial modulation systems with frequency domain equalization, the FFT operations are all done at the receiver: a FFT followed by simple independent equalization of each frequency component, followed by an inverse FFT to restore the equalized serial data stream [SKJ94], [GDC+00], [FAB+02]. As with parallel modulation, transmission is in blocks, separated by cyclic prefixes. In the conventional way of generating these signals, the blocks are sequences of low-pass-filtered coded data symbols, and the cyclic prefixes are formed by appending a copy of the final data symbols at the beginning of the block before filtering. The block and cyclic prefix lengths should obey the same constraints as for parallel modulation. Unlike OFDM, the decoder sees the equivalent of a fixed additive noise channel during a block. Frequency diversity is exploited by the averaging over the frequency band that is inherent in the equalization process.

As for OFDM, zero-padding can replace the CP's [WMS+02]. The need for the $\mathrm{CP}$ can be eliminated in serial modulated systems by transmitting data symbols sequentially, not in blocks, and using overlap-save frequency domain processing at the receiver [FA02].

For both parallel and serial modulation, the received $\mathrm{CP}$ at the beginning of each received block is discarded, and FFT processing is done on each received block. Due to the influence of the transmitted $\mathrm{CP}$, the received block appears to the receiver as if it were one period of a periodic waveform. This fictitious periodic waveform is equivalent to a multicarrier waveform consisting of subcarriers at frequencies which are at multiples of the reciprocal of the period. The multicarrier characteristic is obvious for OFDM and its variants. For serial modulation, the complex-valued samples modulating the virtual multiple subcarriers are revealed by the DFT operation at the receiver.

\section{Generalized Multicarrier Signals}

Figure 1 shows a more general transmitter architecture which includes the aforementioned parallel and serial types of block transmission with cyclic prefixes. The inverse FFT block is 
preceded by a general matrix operation, which may also include spreading, a selection mechanism and/or an allocation to multiple transmitting antennas in a MIMO (multiple input, multiple output) or space-time code. This structure, called "generalized multicarrier CDMA" in [WG00], can be specialized to OFDM, single carrier with frequency domain equalization (SC-FDE), and other frequency domain-based modulation and multiple access schemes. Recognition of this generalized structure can also be found in [FH97], [BR02], [WWN03] and [HPL+04]. We will call the initial matrix block the pre-matrix, and the corresponding class of signals generalized multicarrier (GMC) signals. The GMC architecture provides a general framework for air interface technologies that are applicable to next generation wireless systems. It also suggests a modular signal processing architecture which can be exploited to allow modulation and multiple access schemes to be changed dynamically in response to user requirements and the radio environment.

The structure envisages data being transmitted in blocks, separated by cyclic prefixes, zero-padding or PRP. The organization of data into blocks is carried out by the serial to parallel converter $(\mathrm{S} / \mathrm{P})$. Following the parallel to serial converter $(\mathrm{P} / \mathrm{S})$ after the inverse FFT, time-windowing may be applied to reduce the sidelobes of the transmitted spectrum.

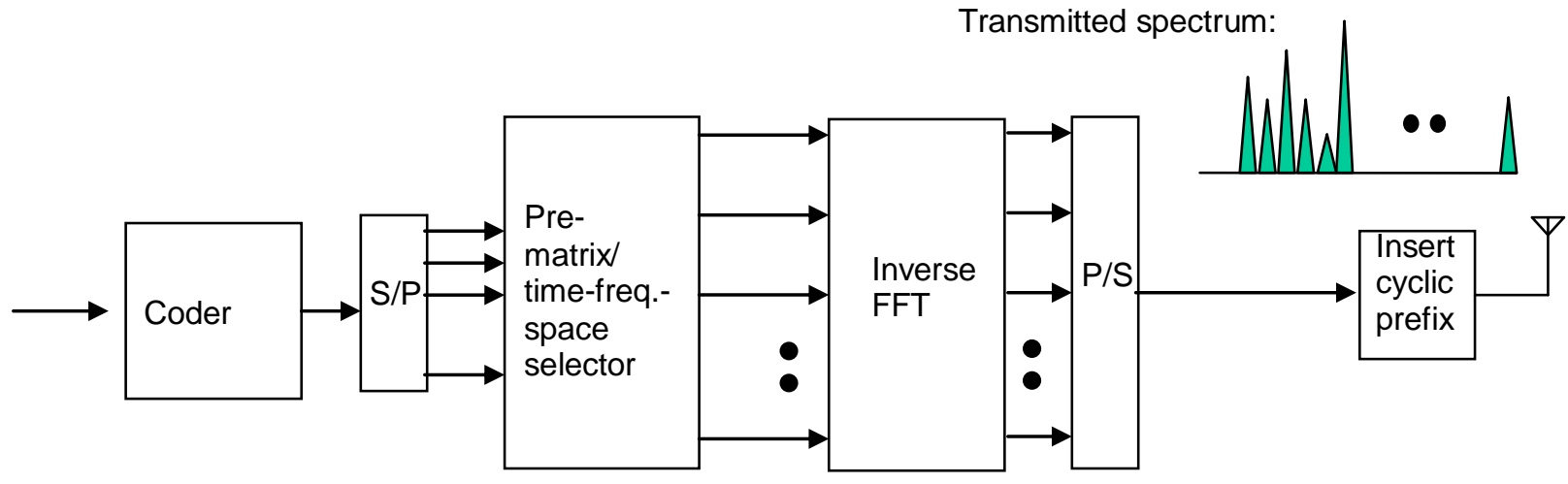

Figure 1 Generalized multicarrier transmitter [FFT means fast Fourier transform operation].
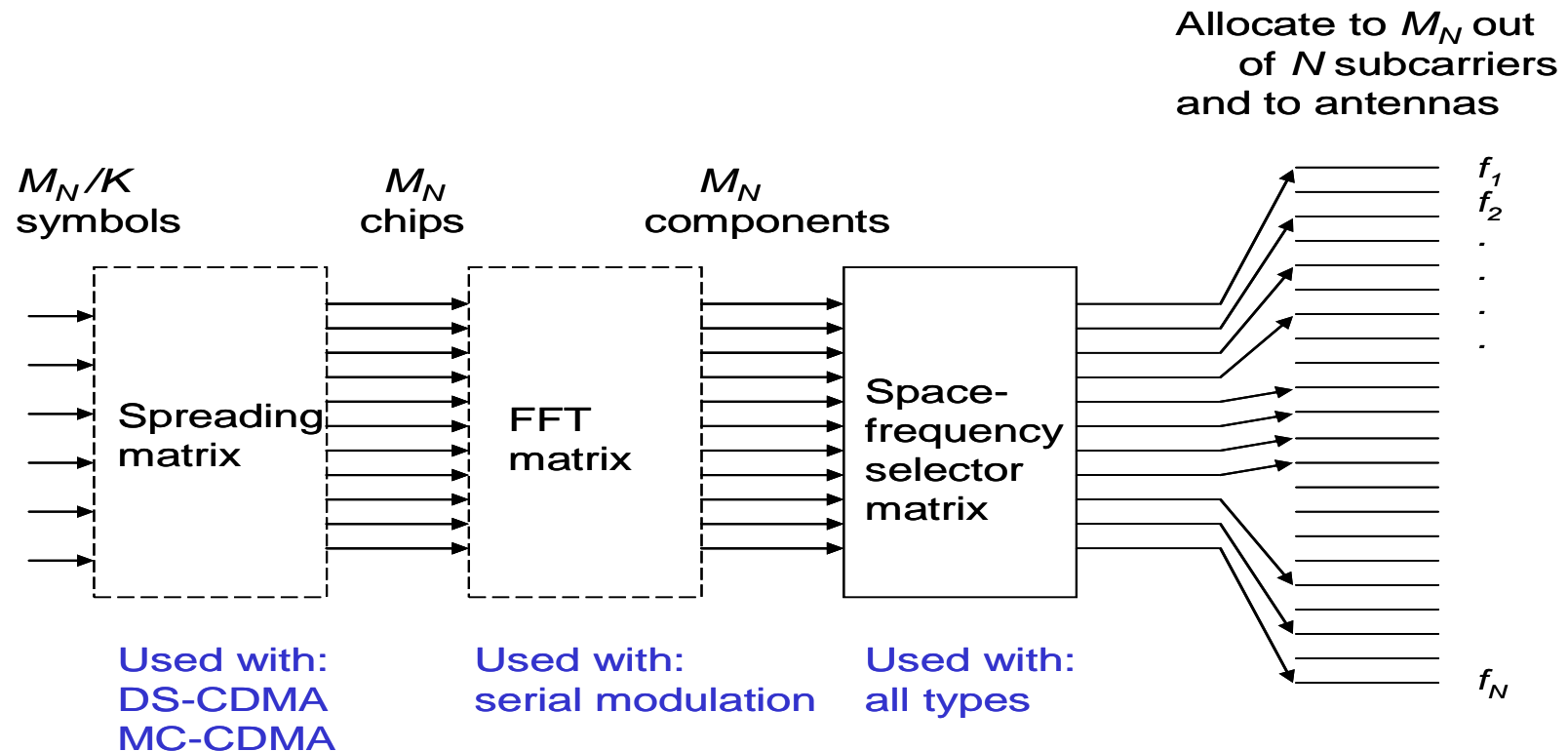

Figure 2 Details of the pre-matrix time-frequency selector in Figure 1 (dashed boxes are optional)

Figure 2 shows the details of the block labeled "matrix timefrequency selector" for several options to be described next. The space-frequency selector matrix maps $M_{N}$ input samples onto a desired set of frequencies, and also, if desired onto different antennas in a MIMO system. It can also include the functions of bit- and power-loading of subcarriers. The FFT matrix is omitted for OFDMA, but is used for serial modulation.

Conventional OFDM results from making the pre-matrix operation a simple identity matrix. A spreading pre-matrix (with 
$M$ input symbols and $M K$ output chips, where $K$ is the spreading factor) produces multicarrier CDMA (MC-CDMA). If the prematrix functions simply as a selector, distributing data symbols onto a number of subcarrier frequencies within a band, the result is an orthogonal frequency division multiple access (OFDMA) signal. In this case, signals to or from different user terminals occupy different segments of the same DFT block, and thus do not interfere with one another. A block of $M_{N}$ coded data symbols $\left\{A_{\ell}, \ell=0,1, \ldots M_{N}-1\right\}$ to or from a particular user is transmitted as the waveform consisting of $N_{c}$ samples

$$
s(k)=\frac{1}{N_{c}} \sum_{\ell=0}^{M_{N}-1} A_{\ell} \exp \left(j \frac{2 \pi k f(\ell)}{N_{c}}\right), \text { for } k=0,1, \ldots N_{c}-1,
$$

where $\left\{f(\ell), \ell=0,1, \ldots M_{N}-1\right\}$ represents a set of $M_{N}$ frequencies to which the data symbols are mapped for that user. In the case of MC-CDMA with a spreading factor of $K$, the $\left\{A_{\ell}\right\}$ are chips representing $M_{N} / K$ data symbols, with $\ell=0,1, \ldots M_{N}-1$, where $M_{N}$ is the number of chips per block.

Low and high bit rate user signals can be multiplexed onto a common sequence of FFT blocks by allocating different numbers of time and frequency slots. FFT block lengths would be long - on the order of thousands of symbols - to accommodate both low and high bit rate users in the same frame.

If the pre-matrix includes a FFT matrix, the resulting transmitted signal is a block serial-modulated single carrier signal. In this case, the coded data symbols are denoted $\{a(m)\}$, and

$$
A(\ell)=\sum_{m=0}^{M_{N}-1} a(m) \exp \left(-j \frac{2 \pi m \ell}{M_{N}}\right)
$$

Substituting (2) into (1), with $f(\ell)=\ell$ results in the traditional form of a serial modulated waveform

$$
s(k)=\sum_{m=0}^{M_{N}-1} a(m) g\left(k-m \frac{N_{c}}{M_{N}}\right)
$$

where $g(n)=\frac{1}{N_{c}} \exp \left(j \frac{\pi}{N_{c}}\left(M_{N}-1\right) n\right) \frac{\sin \left(\frac{\pi M_{N}}{N_{c}} n\right)}{\sin \left(\frac{\pi}{N_{c}} n\right)}$ is a

bandlimited pulse waveform.

Traditionally, serial modulated signals are generated by multiplying a serial train of pulses by data symbols and filtering the result. The potential usefulness of the alternative structure of Figure 1 and equations (1), (2) and (3) to serial modulation comes through additional operations that may be done between the FFT and inverse FFT; examples are frequency domain filtering for spectrum-shaping, and distribution of FFT coefficients to a non-contiguous set of frequencies. Spectrumshaping of both parallel and serial modulated signals can be effected by time-windowing the waveform $s(k)$, or by filtering it.

The subcarrier frequencies $\{f(\ell)\}$ used by any given user may or may not be contiguous. A serial modulation and multiple access scheme sometimes called IFDMA [SDS98] or FDOSS (frequency domain orthogonal signature sequences) can be generated from Figure 1, by mapping $M$ FFT outputs from each of up to $K$ users onto $N_{c}=M K$ frequencies as follows: user $p$ $(p=0,1, \ldots K-1) \quad$ is assigned to the frequency set $\{p, p+K, p+2 K, \ldots p+(M-1) K\}$. Each user thus occupies a unique set of $M$ frequencies in an interleaved fashion; there is no interuser interference, even for frequency selective channels. Furthermore it can be shown [FDL+03], [FDM+03] that the resulting waveform from a given user is equivalent to a serial modulated waveform which results from re-arranging the order of the chips of a direct sequence CDMA (DS-CDMA) waveform with a spreading factor of $K$. It therefore has the low PAPR properties of serial modulated waveforms. This type of signal has been rediscovered and named a number of times. For example: IFDMA (interleaved FDMA) [SDS98], FDOSS (frequency domain orthogonal signature sequences) [CC00],[DFL+04], OFDM-FDMA [GR02], UP-OFDMA (unitary-precoded OFDMA) [XZG03], VSCRF-CDMA (variable spreading and chip repetition factor CDMA) $[\mathrm{GKA}+03],[\mathrm{MKH}+03]$ and carrier interferometry OFDM (CIOFDM) [WWN03]

Reiterating, it is clear that "serial modulation" and "parallel modulation" signals, transmitted in block format, preceded by an adequate cyclic prefix, can be considered as special cases of the GMC signal format. The transmitted signal is equivalent to a sum of discrete frequency components, each modulated by data which have passed through the pre-matrix. The signal is furthermore organized in successive blocks, or frames, each preceded by a cyclic prefix. The main difference between the serial modulation and parallel modulation forms of GMC is in the presence or absence, respectively of the FFT operation at the transmitter in Figure 1. ${ }^{2}$ Their transmitted waveforms differ in that their data symbols are transmitted serially and in parallel, respectively. [WG03] points out that more general complex-field coding operations are possible prior to the transmitter's IFFT operation, to create multicarrier signal classes with greater diversity gains than are possible with pure OFDM. The FFT operation is a special case of such a complex field code. As we point out later, the FFT operation significantly reduces the transmitted signal's instantaneous power dynamic range, and therefore the backoff required for a nonlinear power amplifier.

Figure 3 shows the general receiver structure for GMC signals. The first element is a FFT operation, followed by a selector (or sampler) if necessary, and an equalizing frequency domain filter. For coded OFDM, the function of the equalizing filter can be subsumed within the soft decision decoding effort. The job of the equalizing filter is to scale each frequency component in amplitude and phase, either to completely compensate for the channel's frequency selectivity (as in the case of linear equalization), or to partially compensate for it (as for decision feedback equalization $[\mathrm{FAB}+02],[\mathrm{BT} 02 \mathrm{a}],[\mathrm{TVD}+03])$. The 
linear operation after channel equalization depends on the transmitter's pre-matrix operation: detection and decoding for OFDM or OFDMA, a correlation operation for MC-CDMA, IFFT for serial modulation, etc. All the basic forms of GMC systems offer an overall (transmitter plus receiver) signal processing complexity which is approximately proportional to the logarithm of the maximum channel impulse response length.
Pure time domain equalizer signal processing complexity typically would grow at least linearly or quadratically with impulse response length. For impulse responses spanning more than about 10 or 15 symbols, frequency-domain based systems offer a significant complexity advantage over their time domain counterparts.

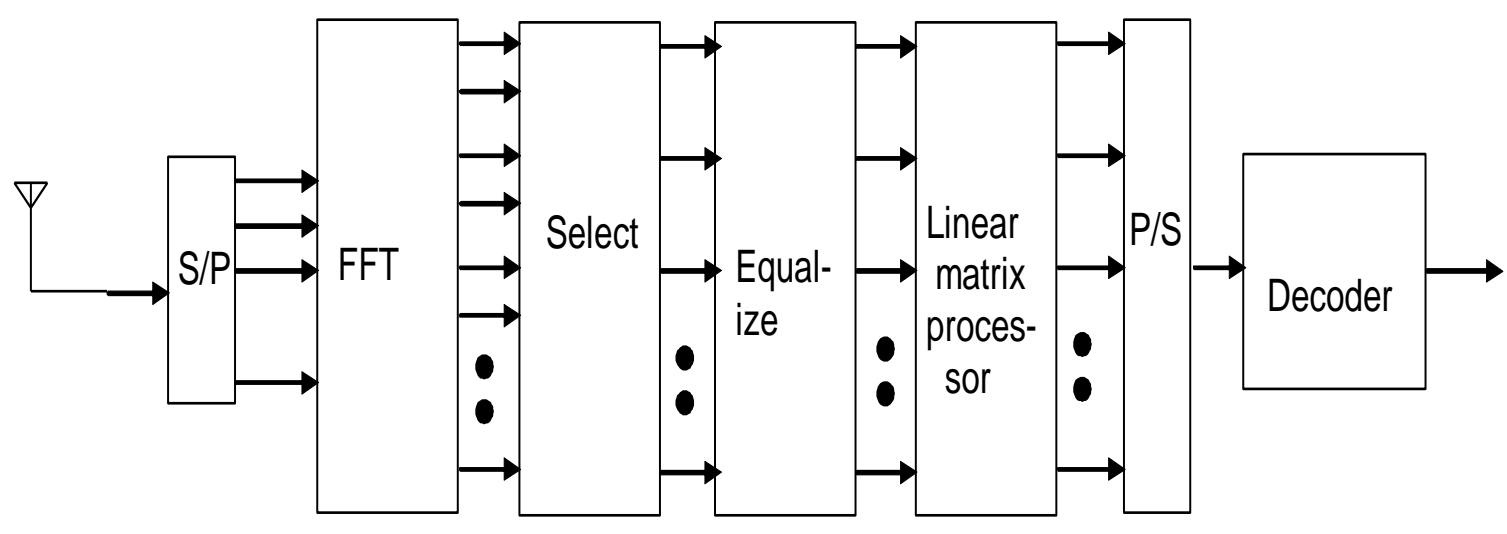

Figure 3 Generalized multicarrier receiver

\section{Bit Error Rate Performance of Parallel and Serial Modulated Systems}

OFDM and OFDMA typically make use of powerful BICM (bitinterleaved coded modulation) schemes [CTB98] that exploit estimated knowledge of the signal to noise ratio at each frequency component. OFDM and OFDMA transmitters can also "adaptively load" subcarriers; i.e. to optimize the allocation of coded bits and power to each subcarrier, according to its channel gain or signal to interference plus noise ratio (SINR). OFDM or OFDMA systems with adaptive loading approximate the optimum "water-filling" prescription for frequency selective channels arrived at from information-theoretic considerations [YLF93],[ZK89] , [FH97], [LVS03]. Adaptive loading requires feedback from the receiving terminal to the transmitter, and accurate tracking of time varying channel frequency responses. For time division duplexing (TDD) systems with slow channel variations, the downlink frequency response is available at the base station from frequency response estimation of the received uplink signal. Given such channel information at the transmitter, adaptive bit loading combined with adaptive coding and power allocation to the subcarriers give OFDM and OFDMA unrivalled flexibility and bit error rate performance on frequency selective channels [WCL+99],[KH97], [KLL03] .

In serial modulated systems, each data symbol is transmitted over the entire signal bandwidth. After equalization, each data symbol in a block thus sees essentially the same signal to noise ratio; the equalized channel appears to the decoder as an additive noise channel with a constant signal to noise ratio which is determined by the channel's fading and frequency selectivity characteristics and the equalizer's compensation of them. A coded modulation scheme tailored to additive white Gaussian noise channels is appropriate, for example pragmatic trellis coded modulation or turbo coding with symbol interleaving which is confined to one block.

Comparisons of coded linear SC-FDE systems with nonadaptively-loaded coded OFDM systems have shown that the two systems offer similar, but not identical, bit error rate performance [SKJ94], [MG01], [FAB+02], for the same average received SNR.

Figure 4, from [MG01], shows the bit error rate performance of coded OFDM and coded single carrier systems with frequency domain linear equalization (SC-FDE) signals over indoor frequency selective Rayleigh-fading channels with a maximum delay spread of $0.66 \mu \mathrm{s}$, corresponding to over 40 coded data symbols, and bit rates from 60 to $300 \mathrm{Mb} / \mathrm{s}$ (depending on the code rate). The SC-FDE systems used trellis coded modulation, while the OFDM systems used BICM. For code rates of about $1 / 2$ or less, non-adaptively-loaded coded OFDM shows a 0.5 to $1 \mathrm{~dB}$ average SNR gain over coded linearly-equalized SC-FDE. Higher code rates tend to favour SC-FDE. Similar conclusions were reached in [WMG04]. Both systems' performance can be further enhanced - for example SC-FDE by the addition of fulllength or sparse $[\mathrm{FAB}+02]$ time domain decision feedback equalization (DFE), iterative equalization [BT02b], [DGE03], [SL03] or turbo equalization [ATM01] [KM04], and OFDM by iterative detection/decoding [LR97], [Kai02]. 


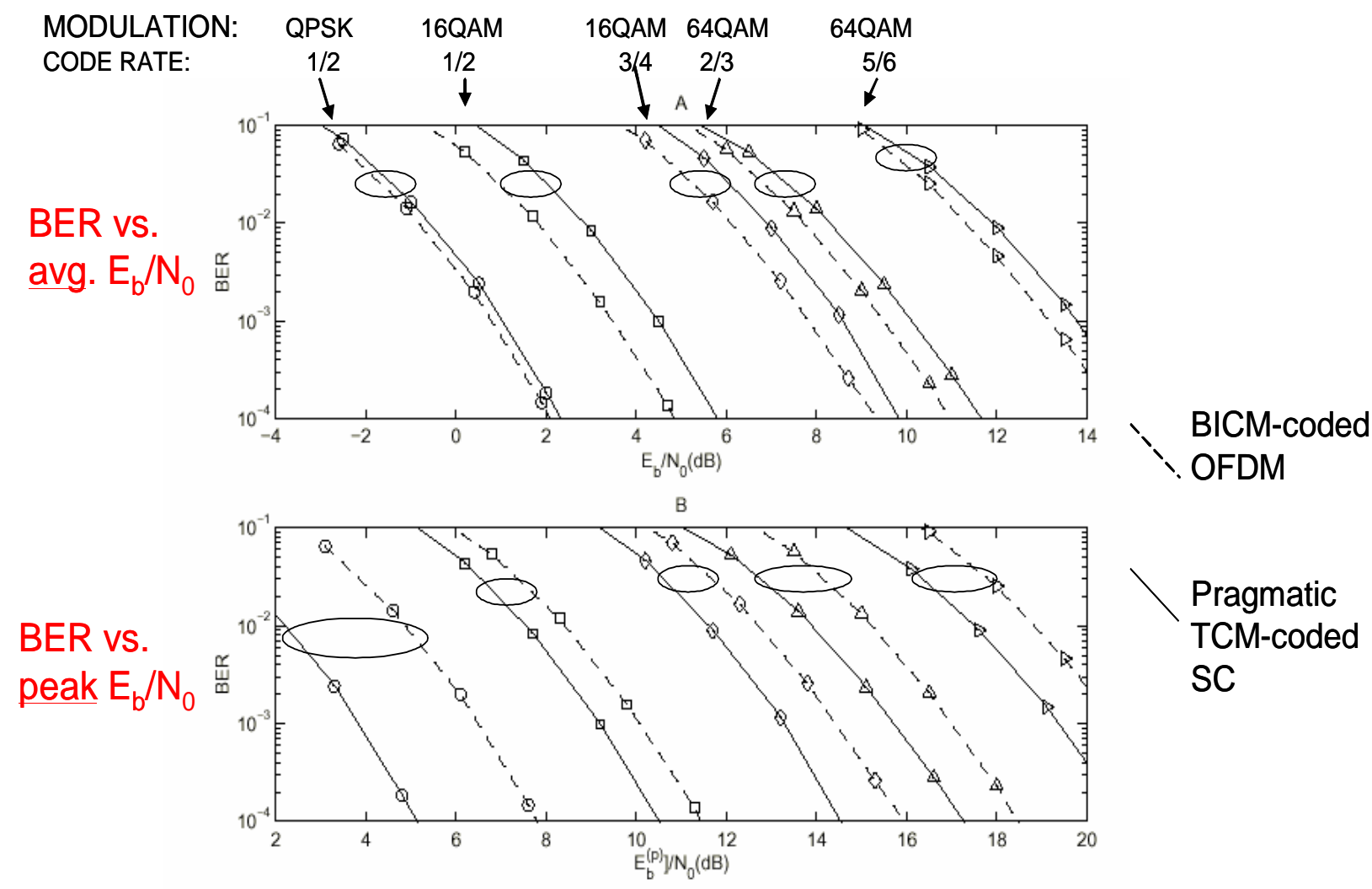

Figure 4 (from [MG01]) Bit error rate performance for pragmatic TCM-coded SC-FDE (solid lines)and BICM-coded OFDM (dashed lines); $\square$ : QPSK, rate $1 / 2 ; \square: 16 \mathrm{QAM}$, rate $1 / 2 ; \diamond: 16 \mathrm{QAM}$, rate $3 / 4 ; \triangle: 64 \mathrm{QAM}$, rate $2 / 3 ; \triangleright: 64 \mathrm{QAM}$, rate $5 / 6$. Part A shows BER versus average $E_{b} / N_{0}$; part B shows BER versus peak $E_{b} / N_{0}$.

Figure 4 also shows that when bit error performance is measured against peak signal to noise ratio (where the peak amplitude per block is averaged over many blocks), SC-FDE is consistently superior to OFDM. In these performance results, the transmitted OFDM signal undergoes clipping and filtering to reduce its peak power. In effect, this comparison is between OFDM and SC systems which are using the same power amplifier with a fixed peak transmitted power output. The worse performance of OFDM is a consequence of the higher peak to average power ratio (PAPR) of the OFDM signal waveform.

\section{Single- and Multi-carrier CDMA}

A spreading pre-matrix (with $M$ input symbols and $M K$ output chips, where $K$ is the spreading factor) produces the multicarrier CDMA (MC-CDMA) form of parallel modulated signals
[FP93], [YLF93], [FK03]. All the chips from a given block of data can be transmitted in one IFFT block, in which case spreading is only in frequency, or they can be distributed among several successive IFFT blocks, in which case spreading is in both time and frequency. The latter case corresponds to signals with both serial and parallel transmission. Different users' data can be multiplexed onto different spreading codes, in which case spreading code chips can occupy both frequency and time dimensions. The serial modulated version of CDMA is direct sequence CDMA (DS-CDMA). It can be generated using the GMC system of Figure 1 and Figure 2 by including the FFT operation as well as the matrix spreading operation. Figure 5 illustrates multi-user OFDMA, OFDM with TDMA, MC-CDMA and FDOSS signals in time, frequency and code dimensions. 
Colors correspond to different uplink-transmitting users.

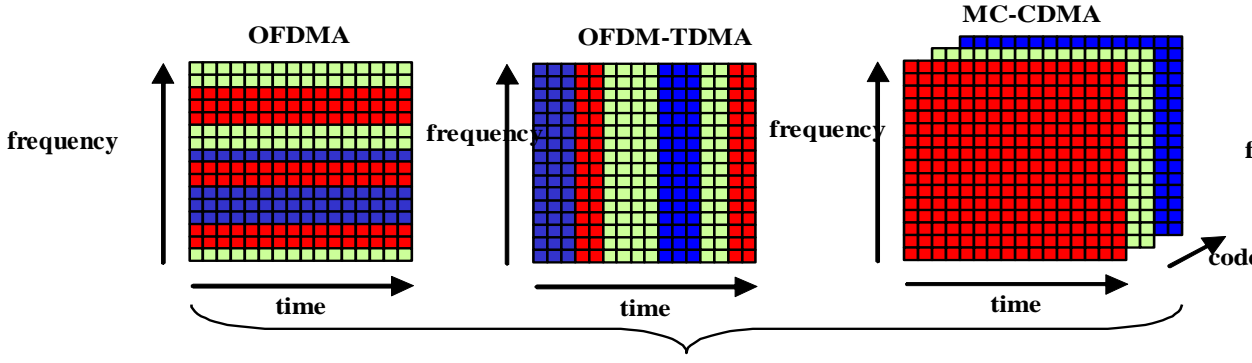

Parallel transmission variants, where each tone is modulated by a coded data symbol

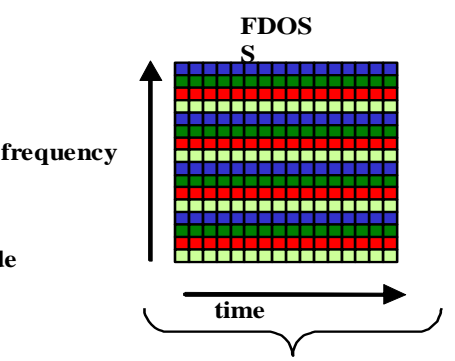

Serial" variant, where each tone is modulated by a FFT coefficient of coded data symbol sequence

Figure 5 Illustrations of multi-user GMC signals: OFDMA, OFDM-TDMA, MC-CDMA, FDOSS

MC-CDMA signals from multiple users with different spreading codes sharing the same frequency and time chip allocations suffer from multiuser interference. They use a frequency domain correlation receiver to suppress but not completely eliminate interference, or a multi-user detector receiver, which can eliminate multiuser interference. If MC-CDMA is used in the downlink path (base station or access point broadcasting to terminals), all the intra-cell interference arrives at a given terminal over a single radio channel; in this case multiuser interference can be removed relatively simply by the use of orthogonal spreading codes and the use of adaptive frequency domain equalization at the receiver, to restore the codes' orthogonality properties. However in the uplink, each user terminal's signal arrives at the base station over a different radio channel, and hence a single equalizer is not valid for all users. Individual frequency domain multiuser detectors can be employed, but their complexity increases significantly with spreading gain or number of interferers.

MC-CDMA systems can be an attractive alternative to pure OFDM systems because they can exploit antenna sectorization and spreading code orthogonality to achieve more efficient frequency reuse in cellular systems, and also because their resistance to multipath is enhanced by their spectrum-spreading [Kai98], [Kai02], [AAS03]. Moreover, MC-CDMA offers efficient multiuser detectors with reasonable complexity compared to DS-CDMA, including iterative Turbo detectors with soft interference cancellation [KH97]. The MC-CDMA signal design allows the spreading factor to be much smaller than the number of subcarriers, which in combination with frequency interleaving gives high diversity gain at reduced receiver complexity. This flexibility is known as "M\&Q" modification [FK03]. However in situations where multiuser intra-cell interference is problematic, systems such as OFDM, OFDMA, SS-MC-MA or FDOSS may be preferable since they avoid multi-user interference $[\mathrm{MKH}+03]$, at the expense of somewhat reduced multipath immunity. MC-CDMA used in the downlink is more robust to multipath than is serial modulated DS-CDMA with RAKE reception [FK03], [AAS+01]. For the uplink of cellular systems, parallel modulated SS-MC-MA or serial modulated DS-CDMA is found to yield better performance than MC-CDMA because of more efficient channel estimation from transmitted pilots [FK03], [AAS+01]. For a fixed bandwidth, each user's spreading factor varies inversely with its bit rate. The DS-CDMA base station receiver could carry out conventional RAKE or correlation processing in the frequency domain to combat multipath and separate different user signals. Higher capacity and spectral efficiency would be achieved by minimum mean squared error (MMSE) multi-user detection using frequency domain processing of the received uplink DS-CDMA signals. Unfortunately the complexity of the MMSE receiver's adaptation processing increases sharply with the spreading factor or number of interfering signals [BCW00], [BR02]. For low bit rate signals, with large spreading factors and numbers of interferers, the MMSE multi-user detection adaptation and complexity would be prohibitive.

Two promising alternative to DS-CDMA in the uplink exist: 1) Use of the FDOSS, or IFDMA signals mentioned earlier. They have the serial modulation, low PAPR and DS-CDMA properties, and have the advantage that multi-user interference is eliminated simply by appropriate sampling of the DFT of the received signal blocks at the receiver. However they have two disadvantages relative to conventional serial DS-CDMA:

- They have a lower diversity order, since a FDOSS signal occupies only the $M$ subcarriers in a block, whereas a DS-CDMA signal occupies up to $M K$, where $M$ is the number of data symbols per block, and $K$ is the spreading factor. FDOSS diversity can be improved by inter-block interleaving and frequencyhopping, at the expense of extra delay. 
- FDOSS signals have no spreading code protection from FDOSS signals in adjacent cells.

In an interference environment, the multiuser interference robustness of FDOSS signals more than compensates for their diversity loss. The problem of interference from adjacent cell uplink FDOSS signals can be solved by optimal spatial combining at the base station and/or by applying direct sequence spreading to the data symbols before FDOSS transmission [GKA+03].

2) SS-MC-MA combines the advantages of OFDM and spectrum spreading. As with FDOSS, multi-user interference is avoided and channel estimation and detection are simplified. The used subcarriers per user are reduced compared to MCCDMA, which reduces the diversity gain. However, due to frequency interleaving, the achievable diversity gain is still the spreading code length. The PAPR is higher in the uplink than with FDOSS but less than with MC-CDMA since fewer subcarriers are used. The resistance to inter-cell interference with SS-MC-MA is comparable to that of MC-CDMA.

\section{Zero Padded OFDM (ZP-OFDM) and Pseudo-Random-Postfix OFDM (PRP- OFDM)}

The ZP-OFDM modulation scheme has been studied in [MWG+02], [Gia97]. The idea is to replace the guard interval sequence of CP-OFDM by a zero padding sequence of identical length. With the same transmission scheme, several different equalization approaches are possible in the receiver [MWG+02] ranging from low complexity/medium performance (OverlapAdd based) to high complexity/high performance (MMSE based equalization). In particular, [MWG+02] demonstrates that (in contrast to CP-OFDM) symbol equalization is possible even if frequency domain channel nulls fall onto data carriers. However, ZP-OFDM still requires channel estimates which are typically obtained by learning symbols and/or pilot tones.

The PRP-OFDM modulation scheme has been studied in $[\mathrm{MCD}+03]$ for the single-antenna context and in $[\mathrm{MDC}+04]$ for the multiple-antennas context. The idea is replace the zerosequence of ZP-OFDM by a pseudo-randomly weighted deterministic sequence [MDC+04]. These weighting factors prevent any signal stationarity and thus spectral peaks. This is desirable, since in the presence of frequency selective fading the spectrum should be as flat as possible - otherwise, the system performance would depend on the band affected by the fading.

[MCD+03] demonstrates that PRP-OFDM keeps all advantages of ZP-OFDM: different equalization approaches are possible in the receiver ranging from low complexity/medium performance (Overlap-Add based) to high complexity/high performance (MMSE based equalization). Also, symbol equalization is possible even if frequency domain channel nulls fall onto data carriers. Additionally to these features, PRP-OFDM allows simple channel estimation and tracking based on the deterministic postfix sequences: A first idea consists in exploiting that the OFDM data symbols are zero mean; a simple mean-value calculation is sufficient to extract the postfix sequence convolved by the channel [MCD+03]. The channel itself is extracted by de-convolution. [MCM+05] demonstrates that such an approach must be refined in practice if higher order constellations are used (QAM64 and higher). Since the postfix sequence and the CP-OFDM guard interval are of same power and duration as the guard interval of CP-OFDM, a higher spectral efficiency can be obtained; in particular, the typical overhead in terms of learning symbols and pilot tones for CPOFDM is avoided.

PRP-OFDM typically is a suitable choice if the target application requires: i) a minimum pilot overhead; ii) lowcomplexity channel tracking (i.e. high mobility context) and iii) adjustable receiver complexity/performance trade-offs (available due to the similarities to ZP-OFDM) without requiring any feedback loop to the transmitter.

\section{OFDM/OffsetQAM (OFDM/OQAM) and IOTA-OFDM}

OFDM/OffsetQAM is an alternative to conventional OFDM modulation. Contrary to it, OFDM/OQAM modulation does not require the use of a guard interval, which leads to a gain in spectral efficiency. Although a guard interval is a simple and efficient way to combat multi-path effects, better performance can be reached by modulating each subcarrier by a prototype function. To obtain the same robustness to the multi-path effects as OFDM with a guard interval, this prototype function must be very well localized in both the time and frequency domains. The localization in time aims at limiting the inter-symbol interference and the localization in frequency aims at limiting the inter-carrier interference (e.g. due to Doppler effects).

The orthogonality between the sub-carriers must also be maintained after the modulation. The optimally localized functions having these properties exist but only guarantee orthogonality on real values. An OFDM modulation using these functions is denoted OFDM/OffsetQAM. We can note that in OFDM/OQAM, each sub-carrier carries a real valued symbol but the density of the sub-carriers in the time-frequency plane is two times greater in OFDM/OQAM than in conventional OFDM, also called OFDM/QAM, with no guard interval.

A particular prototype function called IOTA (Isotropic Orthogonal Transform Algorithm) is discussed in [FAB95] . By construction, the IOTA function has the same shape in both time and frequency domain. We typically call IOTA-OFDM an OFDM/OQAM system using the IOTA function. As stated before, with IOTA-OFDM real valued symbols are transmitted at twice the rate of conventional OFDM in the case of no guard interval inserted between the symbols [JDL+04].

\section{Effect of Phase Noise and Frequency Offsets}

Frequency offset and phase noise are impairments to the received signal that result in inter-carrier interference for parallel modulated systems (OFDM, OFDMA and MC-CDMA) [PVM95], [RK95], [SMS98],[RK99] , [SM01] [TLP00]. These impairments are especially significant for uplink signals from multiple users with different local oscillators. For OFDM, OFDMA and MC-CDMA, the degradation from a frequency offset $\Delta f$ is approximately proportional to $\left(N_{c} \Delta f\right)^{2}$, where $N_{c}$ is the number of subcarriers [PVM95]. Clearly the sensitivity of parallel modulated systems to phase noise and frequency offset 
increases with the FFT block length $N_{c}$. There exist receiver signal processing techniques for OFDM systems to counter frequency offset and phase noise, but they are relatively complicated, since they involve mitigation of frequency domain intersymbol interference [Arm99], [LBM+04].

For serial modulated systems, relatively less degradation results from frequency offset and phase noise (proportional to $\Delta f^{2}$ ) [PVM95]]. In these systems, a constant frequency offset just produces a slowly linearly increasing or decreasing phase shift over the received sequential data symbols. There is negligible additional intersymbol interference. The slow phase shift variation is easily estimated by simple decision-directed techniques, and removed at the receiver prior to detection.

\section{Power Amplifier Efficiency}

Power amplifier efficiency is important since the power amplifier usually constitutes a substantial portion of the cost of a wireless user terminal, and power amplifier cost rises very sharply with peak power rating. Peak output instantaneous power exceeding a power amplifier's linear range causes significant spectral regrowth, and bit error rate (BER) degradation resulting from nonlinear distortion. Since a transmitted parallel modulated signal consists of many parallel modulated subcarriers, it has a significantly higher peak to average power ratio (PAPR) than a comparable serial modulated signal. As a result, parallel modulated systems require several $\mathrm{dB}$ more power backoff and more expensive power amplifiers than do comparable serial modulated systems for the same average power output [SG01], [CND+01]. For given power amplifier the larger the power backoff, the lower the cell coverage. This power backoff penalty is especially important for subscribers near the edge of a cell, with large path loss, where lower-level modulation such as BPSK or QPSK modulation must be used. Recall from Figure 4 that bit error rate performance is evaluated as a function of the peak bit energy to noise ratio, averaged over an ensemble of channels, coded SCFDE is consistently superior to coded OFDM over a wide range of code rates and QAM constellation sizes [MG01]. In effect, this comparison is between parall

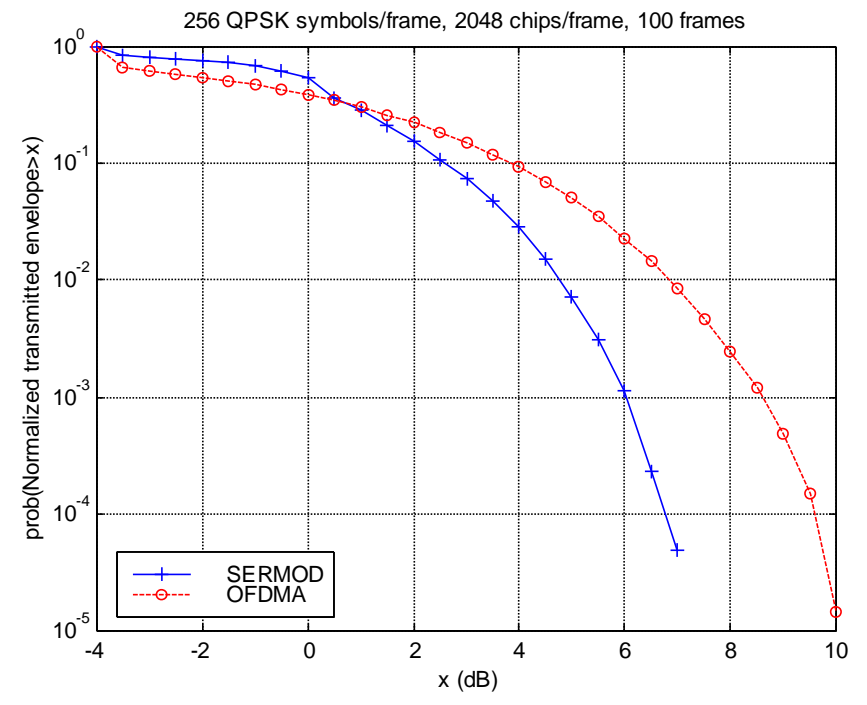

el and serial modulated systems which are using the same power amplifier with a fixed peak transmitted power output.

In practice it is usually found that the power amplifier nonlinearity has a more critical effect on the re-growth of power spectrum sidelobes than on bit error rate performance. In other words, at levels of nonlinearity which significantly affect spectrum sidelobes, the effect on received bit error rate performance is negligible. This is especially relevant when spectral masks permit only very low out of band sidelobes.

Figure 6 shows examples of the instantaneous to average power ratio (IAPR) distributions for single-user OFDMA and serial modulated signals transmitted in the uplink. $N_{c}$ is $2048, M_{N}$ is 256. The waveforms were generated using expression (1). No explicit PAPR-reduction schemes were applied (unless the extra FFT operation in serial modulation is thought of a PAPRreduction scheme for OFDMA). Spectral sidelobes were reduced by applying a $6 \%$ raised cosine time window to each waveform. It is clear that the serial modulated waveform has a significantly lower dynamic range than the corresponding OFDMA waveform. This implies that the serial modulated waveform will require a lower power backoff at the input of a nonlinear transmitter power amplifier. For example, Figure 7 shows the power spectra of the two waveforms at the output of an amplifier whose nonlinear characteristic is modeled by a AM/AM conversion Rapp model [Rap91], with parameter $p=2$. The power backoffs of the two types of waveforms are adjusted to just conform to an ETSI 3GPP power spectrum mask. In this example the serial modulated waveform requires $2.6 \mathrm{~dB}$ less backoff than the OFDMA signal.

Neighbouring user spectra may be received with large power variability due to differing path losses. Avoidance of adjacent channel interference then requires rather stringent spectral masks. For example, allowable interference to adjacentfrequency receivers is usually specified in terms of maximum interference power at a certain difference and at a certain frequency offset from the interferer's carrier [WCA03]. Under typical transmitted power and path loss conditions, this may imply spectral masks with as much as 40 to $60 \mathrm{~dB}$ of attenuation of transmitted spectrum sidelobes at a normalized frequency offset

of

Figure 6 Distribution functions of instantaneous average power ratio (IAPR) of $S=1$ sub-band OFDMA and serial-modulated signals, $N_{r}=2048, M_{N}=256, S=1$. 


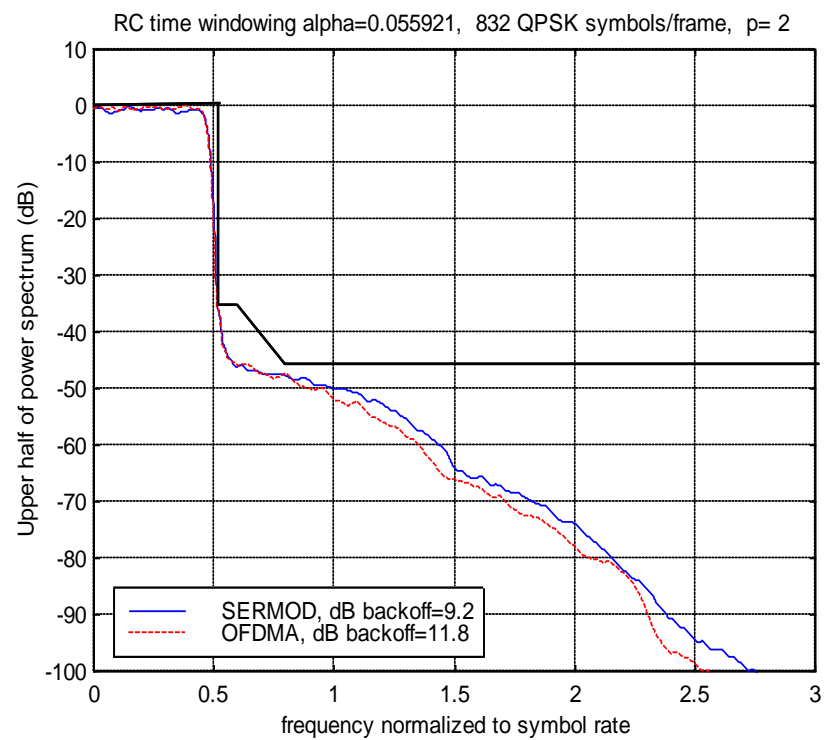

Figure 7 Output power spectra of signals passed through a Rapp model $p=2$ nonlinearity; Backoffs of OFDMA and serial modulated signals are adjusted to produce approximately $-48 \mathrm{~dB}$ relative spectral sidelobe levels. Scaled ETSI 3GPP mask is also shown.

There are signal processing or clipping techniques for reducing the PAPR and spectral regrowth of parallel modulated systems. These include selective mapping and partial transmit sequences [CS00], [MH97a], [MH97b], [ZKT04]; reference signal subtraction [LR99]; coding [JWB94], [JDL+04], [JW96]; and clipping and filtering [Arm02], [DG03], [Och02]. All of these approaches entail extra signal processing complexity, especially at the transmitter; they may also require extra overhead signaling or redundant symbols, and can also degrade performance and bandwidth efficiency [LC98],[VKS01]. It can be shown that for the GMC transmitter of Figure 1, with symbol rate sampling, minimum PAPR is achieved if the pre-matrix and IDFT matrix combine to form an identity matrix. That is, the pre-matrix should be a DFT, and the transmitted signal should be a serial modulated [GR02].

The transmitted serial modulated signal is still in the form of equation (1), and hence can also be considered a "multicarrier" signal. However, as we have seen, using the DFT as in (2) reduces the signal's dynamic range. Thus the DFT operation which creates a serial modulated signal can also be considered as a linear PAPR-reduction operation on an OFDMA signal. Furthermore, it is a very simple operation, in comparison to alternative linear operations such as selective mapping and partial transmit sequences, and requires no side information to be transmitted.

The backoff and power amplifier efficiency are less important issues for the downlink since the cost of the base station power amplifiers is shared among many terminals. Also, since the base station usually has to transmit many signals simultaneously through a common power amplifier, the resulting PAPR will be high regardless of whether individual downlink signals are serial or parallel modulated. However the power amplification issues can significantly influence the cost of the wireless subscriber unit. Thus a very sound air interface approach in cellular systems is to use serial modulation in the uplink (with or without CDMA) and parallel modulation in the downlink [GDC+00], [FAB+02], [FDM+03]. The main virtue of serial modulation for the terminal to base link is the lower peak power of its transmitted waveform and lower required power backoff.

The peak transmitter power requirement of a mobile terminal is also influenced by the multiple access method. Possible uplink multiple access methods, with comparable spectral efficiencies, include TDMA, CDMA, and FDMA, or combinations of them. Pure TDMA requires that all terminals transmit at the same high aggregate bit rate and with the same high peak power. This places a severe cost penalty on terminals that only need to transmit uplink at very low bit rates. CDMA and FDMA are preferable to pure TDMA since they allow each terminal's peak power, as well as its average power, to be proportional to its bit rate.

\section{Spectrum Flexibility}

A useful feature of GMC signals generated as in Figure 1 and Figure 2 is their flexibility to shape the transmitted spectrum to occupy any segment or segments of the frequency band, by means of the selector matrix shown in Figure 2. For example, if the transmitted spectrum is required to fit in a single band of width $M_{N}$, starting at frequency $f_{1}$, the selector distributes the $\left\{A(\ell), \ell=0,1, . . M_{N}-1\right\}$ into the set of frequencies

$$
\Im=\left\{f_{1}+\ell ; \ell=0,1, \ldots M_{N}-1 ;\right\} \text {. }
$$

(4)

Zeroes are inserted into the remaining $N_{c}-M_{N}$ frequencies constituting the block.

If instead, the only vacant spectrum available for transmission of $\left\{A(\ell), \ell=0,1, . . M_{N}-1\right\}$ is in two disjoint parts ("subbands"), denoted respectively $\widetilde{I}_{1}=\left\{f_{1}, f_{1}+1, f_{1}+2, \ldots f_{1}+M_{1}-1\right\} \quad$ and $\mathfrak{I}_{2}=\left\{f_{2}, f_{2}+1, f_{2}+2, \ldots f_{2}+M_{2}-1\right\}$, with bandwidths $M_{1}$ and $M_{2}$ respectively, such that $M_{1}+M_{2}=M_{N}$, the set of data is partitioned into two sets

$a_{1}=\{A(\ell)\}_{\ell=0}^{M M_{1}-1}$ and $a_{2}=\{A(\ell)\}_{\ell=M}^{M} M^{M}, a_{1}$ is mapped onto frequency set $\mathcal{F}_{1}$, and $\boldsymbol{a}_{2}$ is mapped onto frequency set $\mathcal{F}_{2}$. The signal waveform is then generated from the $N_{c}$-point inverse DFT of the resulting block of data symbols and zeros, where we have defined $M_{0}=0$. If more than one user's signal is to be frequency-multiplexed in the downlink, each user's signal would occupy a disjoint set of frequency sub-bands and be generated in this way. This generalizes in an obvious way to more than two sub-bands whose aggregate bandwidth exceeds $M_{N}$. 
Thus a cognitive radio system [FCC03] can characterize potential interference in time and frequency, and dynamically configure its transmitted spectrum by appropriate selection of frequencies to carry the coded data symbols for a given user [HNM+04]. Figure 8 illustrates this spectrum-sharing concept.

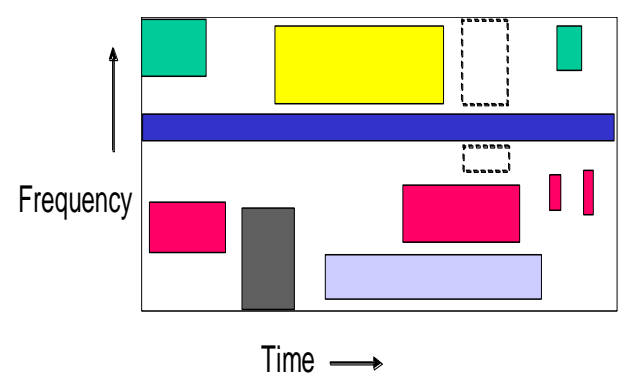

Figure 8 Illustration of spectrum-sharing Each user' is represented by a different colour. A new user's spectrum is tailored to fit into the temporarily unoccupied "white space" denoted by the dashed lines. Such a segmented spectrum can be generated by a suitable time-frequency selector matrix.

If the signal, with cyclic prefix, is considered as being periodic, with period $N_{c}$, it will have a line spectrum

$$
S(f)=\left\{\begin{array}{lr}
A\left(f-f_{i}\right) \text { for } f \in \mathfrak{I}_{i} \\
0 \quad \text { otherwise. }
\end{array}\right.
$$

(5)

The actual transmitted spectrum will be this line spectrum convolved with the $\frac{\sin (\pi f)}{\sin \left(\frac{\pi f}{N_{c}}\right)}$ spectrum of the rectangular time

window of $N_{c}$ samples. The resulting relatively high sidelobes can be suppressed by using a non-rectangular time window on the block plus its cyclic prefix, such as a raised cosine window. Figure 9 shows an example of a multi-band signal's line spectrum, with $S$ sub-bands.

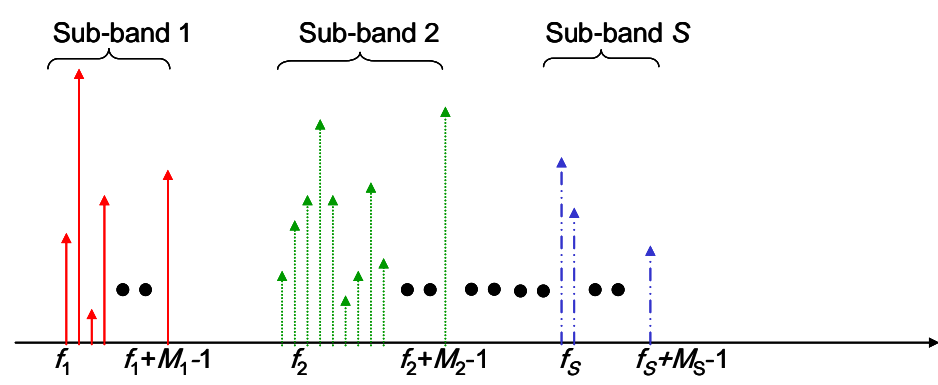

Frequency
For serial modulated signals transmitted over $S$ disjoint subbands, it is straightforward to show from equations (1) and (2) with the above partitioning of $\left\{A(\ell), \ell=0,1, \ldots M_{N}-1\right\}$ and $\mathfrak{I}$, that the counterpart to equation (3) is

$$
s(k)=\frac{1}{N_{c}} \sum_{i=1}^{S} \exp \left(j \frac{2 \pi k f_{i}}{N_{c}}\right) \sum_{m=0}^{M_{N}-1} d_{i}(m) g_{i}\left(k-m \frac{N_{c}}{M_{N}}\right),
$$

where

$d_{i}(m)=a(m) \exp \left(-j \frac{2 \pi m}{M_{N}} \sum_{i^{\prime}=0}^{i-1} M_{i^{\prime}}\right)$ for $m=0,1,2, \ldots M_{N}-1$,

and

$g_{i}(k)=\sum_{n=0}^{M_{i}-1} \exp \left(j \frac{2 \pi n k}{N_{c}}\right)=\exp \left(j \frac{\pi\left(M_{i}-1\right) k}{N_{c}}\right) \frac{\sin \left(\frac{\left.\pi M_{i} k\right)}{N_{c}}\right)}{\sin \left(\frac{\pi k}{N_{c}}\right)}$ for $k=0,1,2, \ldots N_{c}-1$

Expression (7) represents a rotated complex data symbol. Expression Error! Reference source not found.) represents a sampled pulse waveform, similar to a modulated sinc pulse. Thus the waveform expression (6) represents the sum of $S$ complex single carrier waveforms, up-converted by the frequencies $\left\{f_{i}\right\}$. For relatively small values of $S$, like 2 or 3 , its peak to average power ratio will be significantly less than that of a corresponding multi-band OFDMA signal, which is the sum of $M_{N}$ complex exponential waveforms.

For both OFDMA and serial modulation, essentially the same multi-band average power spectra will be produced. Figure 10 illustrates the average power spectra for serial modulation and OFDMA for two sub-bands, with $M_{N}=256$ data symbols within a total bandwidth of $N_{c}=2048$. The other parameters for this figure are $f_{1}=589, M_{1}=103, f_{2}=1390$ and $M_{2}=M_{N}-M_{1}=153$. Note that the OFDMA and serial modulation spectra are almost identical.

Figure 9 Illustration of a multi-band line spectrum 
256 QPSK symbols/frame, 2048 chips/frame, 10 frames, Backoff= $100 \mathrm{~dB}$

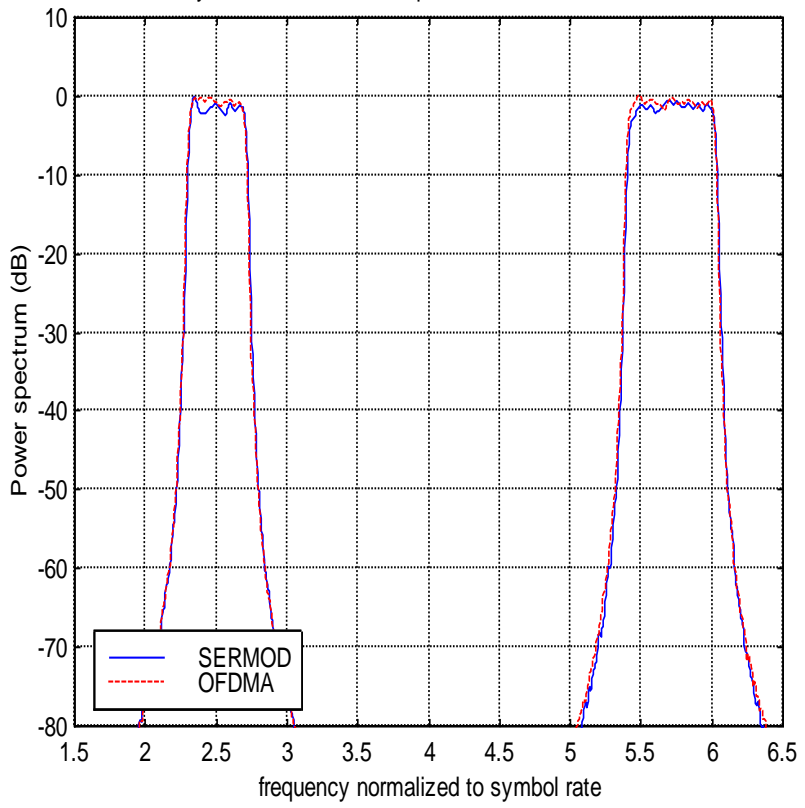

Figure 10 Power spectra for $S=2, N_{c}=2048, M_{N}=256$ for OFDMA and serial modulation

The spectrum flexibility feature of GMC signals also allows a base station to transmit to low cost, low data rate user terminals that have limited receiver bandwidths. Such a terminal would use the same DFT block duration as that of the transmitted signal, but extracts from it, equalizes and decodes only the portion of the frequencies in the block that are relevant to it.

\section{Some Issues for Further Research}

The impact of the proposed mixed mode on the MAC (medium access control) layer and resource allocation needs to be investigated. For example, feedback information must be exchanged for adaptive modulation, power control, synchronization, parameter control for coding, modulation and antennas. Mobile systems' MAC should be modified to take into account the higher expected mobility and rate of channel change in future mobile broadband systems. Also a more detailed study should be made of means to accommodate, within a common allocated frequency band, user terminals with vastly different bit rates; e.g. many small embedded devices sporadically transmitting and receiving say a few hundred bits per second, coexisting with 1,10 and $100 \mathrm{Mb} / \mathrm{s}$ terminals.

Efficient frequency domain parameter estimation methods using training words and/or pilot tones are known for OFDM [HKR97], [LCS98] , [Li02] and SC-FDE systems $[\mathrm{FAB}+02]$.However high bit rates and multipath delay spreads call for large FFT block lengths; there is then a question of the ability of receivers to estimate and track the resulting large number of frequency domain parameters and Doppler shifts over rapidly time varying channels. Investigations should include adaptation using training and blind adaptation as well as the use of unique words (UW) for channel tracking. Since the UW is known at the receiver it can effectively be used for tasks like synchronization (carrier frequency, clock frequency, etc.), channel estimation, and preventing the error propagation in the DFE [TVD+03], [WMS+02]. The CP can be replaced by a short time domain training sequence, which is processed and extrapolated in the frequency domain to estimate the required equalizer frequency domain parameters [FAB+02], [WMS+02].

In this document we have focused on block transmission schemes using frequency domain processing at the transmitter or receiver, and with cyclic prefixes at the beginning of each block, to avoid inter-block interference and to simplify frequency domain filtering with DFT's. Other frequency domain processing methods can be used for systems which do not organize data in blocks separated by cyclic prefixes. The motivation is to eliminate the $\mathrm{CP}$ overhead or to achieve compatibility with legacy systems which did not use CP's. For serial modulated SC-FDE systems, overlap-save processing can be used [FA02]. OFDM systems without the CP have also been proposed [LGA01].

\section{Summary and Recommendations}

We have proposed generalized multicarrier (GMC) signaling as a common framework for future generation wireless systems which must accommodate a very wide range of user bit rates with low cost and high reliability in a difficult, spectrally crowded radio environment. Within this framework, adaptive, reconfigurable transmission techniques are desirable, including not only power and bit rate, but also spectrum occupancy and the choice of the modulation and multiple access/multiplexing scheme itself. For example, downlink parallel modulation together with uplink serial modulation is a good configuration when user terminal power efficiency is a major issue. Flexibility for multi-band operation is also relatively easy to achieve for GMC signaling.

The proposed GMC modes are quite compatible with MIMO and space diversity, and also with spectrum spreading. A number of variations of OFDM and SC-FDE are useful, such as OFDMA, MC-CDMA and FDOSS.

\section{REFERENCES}

[1] [AAS+01] S. Abeta, H. Atarashi, M. Sawahashi and F. Adachi, "Performance of Coherent Multi-Carrier/DSCDMA and MC-CDMA for Broadband Packet Wireless Access", IEICE Trans. Commun., Vol. E84B, No. 3, Mar., 2001, pp. 406-414.

[2] [AAS03] H. Atarashi, S. Abeta and M. Sawahashi, "Variable Spreading Factor-Orthogonal Frequency and Code Division Multiplexing (VSF-OFCDM) for Broadband Packet Wireless Access", IEICE Trans. Commun., Vol. E86-B, No. 1, Jan., 2003, pp. 291-299.

[3] [Arm99] J. Armstrong, "Analysis of New and Existing Methods of Reducing Intercarrier Interference Due to Carrier Frequency Offset in OFDM", IEEE Trans. Communications, Vol. 47, No. 3, March 1999, pp. 365-369.

[4] [Arm02] J. Armstrong, "Peak-to-average power reduction for OFDM by repeated clipping and frequency domain filtering", Electronics letters, 28 Feb., 2002, pp. 246-247. 
[5] [ATM01] $\mathrm{T}$ Abe, $\mathrm{S}$ Tomisato, $\mathrm{T}$ Matsumoto, "Performance Evaluations of a space-time Turbo Equalizer in Frequency Selective MIMO Channels Using Field Measurement Data", IEE Workshop on MIMO Communication Systems, London, Dec., 2001, pp. $21 / 1-21 / 5$.

[6] [BCW00] R.M. Buehrer, N.S. Correal, and B.D. Woerner, "Simulation Comparison of Multiuser Receivers for Cellular CDMA", IEEE Trans. Vehic. Technol., Vol. 49, No. 4, July 2000, pp. 1065-1085.

[7] [BR02] L. Brühl and B. Rembold, "Unified SpatioTemporal Frequency Domain Equalization for Multiand Single-Carrier CDMA Systems", Proc. VTC 2002 fall, Oct. 2002, pp. 676-680.

[8] [BT02a] N. Benvenuto and S. Tomasin, "On the comparison Between OFDM and Single Carrier with a DFE Using a frequency Domain Feedforward Filter", IEEE Trans. Communications, Vol. 50, No. 6, June, 2002, pp. 947-955.

[9] [BT02b] N. Benvenuto and S. Tomasin, "Block Iterative DFE for Single Carrier Modulation", IEE Electronic Letters, Vol. 39, No. 19, Sept. 2002.

[10] [CC00] C-M Chang and K-C Chen, "FrequencyDomain Approach to Multiuser Detection in DSCDMA Communications", IEEE Comm. Letters, Vol. 4, No. 11, Nov., 2000, pp. 331-333.

[11] [CND+01] G. Carron, R. Ness, L. Deneire, L. Van der Perre, and M. Engels, "Comparison of Two Modulation Techniques Using Frequency Domain Processing for In-House Networks", IEEE Trans. Consumer Electronics, Vol. 47, No. 1, Feb. 2001, pp. 63-72.

[12] [CS00] L.J. Cimini, Jr., and N.R. Sollenberger, ’Peakto-average power ratio reduction of an OFDM signal using partial transmit sequences", IEEE Comm. Letters, Vol. 4, No. 3, March, 2000, pp. 86-88.

[13] [CTB98] G. Caire, G. Taricco and E. Biglieri, "BitInterleaved Coded Modulation", IEEE Trans. Information Theory, May 1998, pp. 927-946.

[14] [DFL+04] R. Dinis, D. Falconer, C.T. Lam and M. Sabbaghian, "A Multiple Access Scheme for the Uplink of Broadband Wireless Systems", Proc. Globecom, 2004, Dallas, Dec. 2004.

[15] [DG03] R. Dinis and A, Gusmão, "A Class of Nonlinear Signal Processing Schemes for BandwidthEfficient OFDM Transmission with Low Envelope Fluctuation", IEEE Trans. Communications, Vol. 52, No. 11, Nov., 2004, pp. 2009-2018.

[16] [DGE03] R. Dinis, A. Gusmão, and N. Esteves, "On Broadband Block Transmission over Strongly Frequency-Selective fading Channels", Proc. Wireless 2003, Calgary, July, 2003.

[17] [FA02] D. Falconer and S.L. Ariyavisitakul, "Broadband Wireless Using Single Carrier and Frequency Domain Equalization", Proc. $5^{\text {th }}$ Int. Symposium on Wireless Personal Multimedia Communications, Honolulu, Oct. 27-30, 2002.

[18] [FAB95] B. Le Floch, M. Alard and C. Berrou, "Coded Orthogonal Frequency Division Multiplex", Proc. IEEE, Vol. 83, No. 6, June, 1995.

[19] [FAB+02] D. Falconer, S.L. Ariyavisitakul, A. Benyamin-Seeyar and B. Eidson, "Frequency Domain
Equalization for Single-Carrier Broadband Wireless Systems", IEEE Communications Magazine, Vol. 40, No. 4, April 2002, pp. 58-66.

[20] [FCC03] FCC web site on cognitive radio: www.fcc.gov/oet/cognitiveradio/

[21] [FDL+03] D. Falconer, R. Dinis, C.T. Lam and M. Sabbaghian, "Frequency Domain Orthogonal Signature Sequences (FDOSS) for Uplink DSCDMA", presentation at WWRF meeting \#10, New York, Oct. 2003.

[22] [FDM+03] D. Falconer, R. Dinis, T. Matsumoto, M. Ran, A. Springer and P. Zhu, "WWRF/WG4/Subgroup on New Air Interfaces White Paper: A Mixed Single Carrier/OFDM Air Interface for Future-Generation Cellular Wireless Systems", Aug., 2003.

[23] [Fre05] P. Frenger, "A Framework for Future Wireless Access", Proc. WWRF13, Working Group 4, Jeju, Korea, March, 2005.

[24] [FH97] R.F.H. Fischer and J.B. Huber, "On the Equivalence of Single- and Multicarrier Modulation: A New View", Proc. ISIT 1997, Ulm, Germany, July, 1997.

[25] [FK03] K. Fazel and S. Kaiser, Multi-Carrier and Spread Spectrum Systems, John Wiley and Sons, 2003.

[26] [FK04] D. Falconer and S. Kaiser, "Broadband frequency Domain-based Air Interfaces", presentation at WWRF meeting \#11, Oslo, June, 2004.

[27] [FP93] K. Fazel and L. Papke,"'On the performance of convolutionally-coded CDMA/OFDM," Proc. PIMRC '93, Yokohama, Sept. 1993, pp. 468-472.

[28] [GDC+00] A. Gusmão, R. Dinis, J. Conceição, and N. Esteves, "Comparison of Two Modulation Choices for Broadband Wireless Communications", Proc. VTC 2000 Spring, Tokyo, May 2000, Vol. 2, pp. 13001305.

[29] [Gia97] G.B. Giannakis, "Filterbanks for Blind Channel Identification and Equalization", IEEE Sig. Processing Letters, June 1997, pp. 184-187.

[30] [GKA+03] Y. Goto, T. Kawamura, H. Atarashi and M. Sawahashi, "Variable Spreading and Chip repetition Factors (VSCRF)-CDMA in Reverse Link for Broadband Wireless Access", Proc. PIMRC 2003, Beijing, 2003.

[31] [GR02] D. Galda and H. Rohling, "A Low Complexity Transmitter Structure for OFDM-FDMA Uplink Systems", Proc. VTC Spring 2002, pp. 17371741.

[32] [HKR97] P. Höher, S. Kaiser, and P. Robertson, "Pilot-Symbol-Aided Channel Estimation in Time and Grequency," Proc. GLOBECOM '97 (CTMC), Phoenix, Nov. 1997.

[33] [HNM+04] S. Hijazi, B. Natarajan, M. Michelini, Z. $\mathrm{Wu}$ and C.R. Nassar, "Flexible Spectrum Use and Better Coexistence at the Physical Layer of Future Wireless Systems via a Multicarrier Platform", IEEE Wireless Communications, April 2004, pp. 64-71.

[34] [HPL+04] F. Horlin, F. Petré, E. Lopez-Estraviz, F. Naessens and L. Van der Perre, "A Generic Transmission Scheme for Fourth Generation Wireless Systems", presentation at WWRF meeting \#11, Oslo, June, 2004. 
[35] [ITU03] "Framework and Overall Objectives of the Future Development of IMT-2000 and Systems Beyond IMT-2000", ITU Recommendation ITU-R M.1645, 2003.

[36] [JDL+04] J.P. Javaudin, C. Dubuc, D. Lacroix, and M. Earnshaw, "An OFDM Evolution to the UMTS High Speed Downlink Packet Access", Proc. VTC 2004 fall, Los Angeles, Sept., 2004.

[37] [JW96] A.E. Jones and T. Wilkinson, "Combined coding for error control and increased robustness to system nonlinearities in OFDM", Proc. VTC '96, Atlanta, May, 1996.

[38] [JWB94] A.E. Jones, T.A. Wilkinson and S.K. Barton, "Block coding scheme for reduction of peak to mean envelope power ratio of multicarrier transmission scheme", Electronics Letters, Vol. 30, No. 25, Dec. 1994, pp. 2098-2099.

[39] [Kai98] S. Kaiser, "MC-FDMA and MC-TDMA versus MC-CDMA and SS-MC-MA: Performance Evaluation for Fading Channels", Proc. $5^{\text {th }}$ Int. Symp. On Spread Spectrum Techniques and Applications", Vol. I, 1998.

[40] [Kai02] S. Kaiser, "OFDM Code Division Multiplexing in Fading Channels," IEEE Transactions on Communications, Vol. 50, No. 8, August 2002, pp. 1266-1273.

[41] [KB02] S. Kaiser et al, "WWRF/WG4/Subgroup on New Air Interfaces White Paper: New Air Interface Technologies - Requirements and Solutions", draft version, August, 2002.

[42] [KH97] S. Kaiser and J. Hagenauer, "Multi-Carrier CDMA with iterative decoding and soft-interference cancellation," Proc. GLOBECOM '97, Phoenix, Nov. 1997.

[43] [KLL03] D. Kivanc, G. Li and H. Liu, "Computationally Efficient Bandwidth Allocation and Power Control for OFDMA", IEEE Trans. On Wireless Communications, Vol. 2, No. 6, Nov. 2003, pp. 1150-1158.

[44] [KM04] K. Kansanen and T. Matsumoto, "Frequency Domain Turbo Equalization for Broadband MIMO Channels", presentation at COST 273 meeting, TD(04) 143, Göteborg, June 9-10, 2004.

[45] [KR02] I. Koffman and V. Roman, "Broadband Wireless Access Solutions Based on OFDM Access in IEEE 802.16", IEEE Communications Magazine, Vol. 40, No. 4, April 2002, pp. 96-103.

[46] [KS02] S. Kaiser et al, "White Paper: Broadband Multi-Carrier Based Air Interface", Draft Version 1.0, May 6, 2002.

[47] [LBM+04] G. Leus, I.Barhumi, O. Rousseaux and M. Moonen, "Direct semi-blind design of serial linear equalizers for doubly-selective channels", Proc. 2004 IEEE International Conference on Communications, Volume 5, 20-24 June 2004, pp. 2626 - 2630.

[48] [LC98] X. Li and L.J. Cimini, Jr., "Effects of Clipping and Filtering on the Performance of OFDM", IEEE Communications Letters, Vol. 2, No. 5, May 1998, pp. 131-133.

[49] [LCS98] Y. Li, L.J. Cimini, and N.R. Sollenberger. Robust Channel Estimation for OFDM Systems with Rapid Dispersive Fading Channels. IEEE
Transactions on Communications, 46(7):902-915, July 1998.

[50] [LGA01] D. Lacroix, N. Goudard, and M. Alard, "OFDM with Guard Interval versus OFDM/Offset QAM for High Data Rate UMTS Downlink Transmission", Proc. VTC 2001, fall, Atlantic City, Oct., 2001, pp. 2682-2686.

[51] [Li02] Y. Li, "Simplified Channel Estimation for OFDM Systems with Multiple Antennas", IEEE Trans. Commun., Vol. 50, No. 1, Jan., 2002, pp. 6775.

[52] [LJG02] D. Lacroix, J.P. Javaudin, and N. Goudard, "IOTA, an Advanced OFDM Modulation for Future Broadband Physical Layers", Wireless world Research Forum (WWRF) meeting \#7, Dec. 2002.

[53] [LR97] X. Li and J. Ritcey, "Bit-Interleaved Coded Modulation with Iterative Decoding", IEEE Trans. Communications, Vol. 45, No. 11, Nov. 1997.

[54] [LR99] M. Lampe and H. Rohling, "Reducing out-ofband emissions due to nonlinearities in OFDM systems", Proc. VTC Spring '99.

[55] [LVS03] J. Louveaux, L. Vandendorpe and $T$. Sartenaer, "Cyclic Prefixed Single Carrier and Multicarrier Transmission: Bit Rate Comparison", IEEE Commun. Letters, Vol. 7, No. 4, April 2003 , pp.180-182.

[56] [MCD+03] M. Muck, M. de Courville, M. Debbah and P. Duhamel, "A Pseudo Random Postfix OFDM Modulator and Inherent Channel Estimation Techniques", Proc. Globecom 2003, Dec., 2003.

[57] [MCD04] M. Muck, M. De Courville and P. Duhamel, "Postfix Design for Pseudo Random Postfix OFDM Modulators", Proc. Int. OFDM Workshop, Dresden, Sept. 2004.

[58] [MCM+05] M. Muck, M. De Courville, X. Miet, and P. Duhamel, "Iterative Interference Suppression for Pseudo Random Postfix OFDM Based Channel estimation", IEEE Int. Conf. on Acoustics, Speech and Signal Processing, March 2005.

[59] [MDC+04] M. Muck, A. R. Dias, M. De Courville and P. Duhamel, "A Pseudo Random Postfix OFDMBased Modulator for Multiple Antennae Systems", Proc. ICC 2004, Paris, May, 2004.

[60] [MDD+00] B. Muquet, M. de Courville, P. Duhamel and G. Giannakis, "OFDM with Trailing Zeros Versus OFDM with Cyclic Prefix: Links, Comparisons and Application to the Hiperlan/2 System", Proc. ICC 2000, New Orleans, June, 2000, pp. 1049-1053.

[61] [MG01] P. Montezuma and A. Gusmão, "A Pragmatic Coded Modulation Choice for Future Broadband Wireless Communications", Proc. VTC 2001 Spring, Rhodes, May 2001, Vol. 2, pp. 1324-1328.

[62] [MH97a] S.H. Müller and J.B. Huber, "OFDM with reduced peak-to-average power ratio by optimum combination of partial transmit sequences", Electronics Letters, $27^{\text {th }}$ Feb., 1997, pp. 368-369.

[63] [MH97b] S.H. Müller and J.B. Huber, "A comparison of peak power reduction schemes for OFDM", Proc. Globecom '97.

[64] [MKH+03] N. Maeda, Y. Kishiyama, K. Higuchi, H. Atarashi and M. Sawahashi, "Experimental Evaluation of Throughput Performance in Broadband Packet 
Wireless Access Based on VSF-OFCDM and VSFCDMA", Proc. PIMRC 2003, Beijing, 2003.

[65] [MLM02] W. Mohr, R. Lüder amd K-H. Möhrmann, "Data rate estimates, range calculations and spectrum demand for new elements of systems beyond IMT2000", Proc. $5^{\text {th }}$ Symp. Wireless Personal Multimedia Communications WPMC02, Honolulu Hawaii, USA, Oct. 2002, Vol. 1, pp. 27-30.

[66] [MWG+02] B. Muquet, Z. Wang, G.B. Giannakis, M. De Courville, and P. Duhamel, "Cyclic Prefixing or Zero Padding for Wireless Multicarrier Transmissions?", IEEE Trans. Commun., Vol. 50, No. 12, Dec. 2002.

[67] [Och02] H. Ochiai, "Power efficiency comparison of OFDM and single-carrier signals", Proc. VTC, Fall 2002, pp. 899-903.

[68] [PVM95] T. Pollet, M. Van Bladel and $M$. Moeneclaey, "BER Sensitivity of OFDM Systems to Carrier Frequency Offset and Wiener Phase Noise", IEEE Trans. Communications, Vol. 43, No. 2/3/4, Feb./Mar./April 1995, pp. 191-193.

[69] [Rap91] C. Rapp, "Effects of HPA Nonlinearity on a 4DPSK/OFDM Signal for a Digital Sound Broadcasting System", Proc. $2^{\text {nd }}$ European Conf. on Satellite Communications, Liege, Oct. 1991, pp. 179184.

[70] [RK95] P. Robertson and S. Kaiser, "Analysis of the effects of phase-noise in orthogonal frequency division multiplex (OFDM) systems," Proc. ICC '95, Seattle, June 1995, pp. 1652-1657.

[71] [RK99] P. Robertson and S. Kaiser, "The Effects of Doppler Spreads in OFDM(A) Mobile Radio Systems", Proc. VTC 99, pp. 329-333.

[72] [SDS98] U. Sorger, I. De Broeck and M. Schnell, "Interleaved FDMA - A New Spread-Spectrum Multiple-Access Scheme", Proc. ICC98, Atlanta, June, 1998, pp. 1013-1017.

[73] [SG01] P. Struhsaker and K. Griffin, "Analysis of PHY Waveform Peak to Mean Ratio and Impact on RF Amplification", contribution to IEEE 802.16.3c-01/46, Mar. 6, 2001.

[74] [SKJ94] H. Sari, G. Karam and I. Jeanclaude, "Frequency-Domain equalization of Mobile Radio and Terrestrial Broadcast Channels", Proc. Globecom '94, San Francisco, Nov.-Dec. 1994, pp. 1-5.

[75] [SL03] P. Schniter and H. Liu, "Iterative Equalization for Single Carrier Cyclic Prefix in Doubly Dispersive Channels", Conf. Record Asilomar Conf. on Signals, Systems and Computers, Nov., 2003.

[76] [SLK97] H. Sari, Y. Levy, G. Karam, "An Analysis of Orthogonal Frequency-Division Multiple Access", Proc. of IEEE GLOBECOM'97, pp 1635-1639

[77] [SM01] H. Steendam and M. Moeneclaey, "The Effect of Carrier Frequency Offsets on Downlink and Uplink MC-DS-CDMA", IEEE Sel. Areas in communications, Vol. 19, No. 12, Dec., 2001, pp. 2528-2536.

[78] [SMS98] H. Steendam, M. Moeneclaey, and H. Sari, "The Effect of Carrier Phase Jitter on the Performance of Orthogonal Frequency-Division Multiple-access Systems", IEEE Trans. Communications, Vol. 46, No. 4, April 1998, pp. 456-459.
[79] [TLP00] A.M. Tonello, N. Laurenti, and S. Pupolin, "Analysis of the Uplink of an Asynchronous Multiuser DMT OFDMA System Impaired by Time Offsets, Frequency Offsets and Multipath Fading", Proc. VTC 2000 Fall, pp. 1094-1099.

[80] [TVD+03] J. Tubbax, L. Van der Perre, S. Donnay and M. Engels, "Single Carrier Communications Using Decision-Feedback Equalization for Multiple Antennas", Proc. ICC 2003, anchorage, May 2003, Vol. 4, pp. 2321-2325.

[81] [VKS01] C. Van den Bos, M.H.L. Kouwenhoven,and W. A. Serdijn, "Effect of Smooth Nonlinear Distortion on OFDM Symbol Error Rate", IEEE Trans. Communications, Vol. 49, No. 9, Sept. 2001, pp. 1510-1514.

[82] [VP00] R. van Nee and R. Prasad, OFDM for Wireless Multimedia Communications, Artech House, 2000.

[83] [WCA03] "WCA Coalition Proposal to the FCC in the Matter of Amendment of Parts 1,21, 73, 74, and 101 of the Commission's Rules to Facilitate the Provision of Fixed and Mobile Broadband Access, Educational and Other Advanced Services in the 2150-2162 and 2500$2690 \mathrm{MHz}$ Bands", Sept. 8, 2003.

[84] [WCL+99] C.Y. Wong, R.S. Cheng, K. B. Letaief and R.D. Murch, "Multiuser OFDM with Adaptive Subcarrier, Bit and Power Allocation", IEEE Sel. Areas in Communication, Vol. 17, No. 10, Oct., 1999, pp. $1747-1758$.

[85] [WG00] Z. Wang and G.B. Giannakis, "Wireless Multicarrier Communications - Where Fourier Meets Shannon", IEEE Sig. Proc. Mag, May, 2000, pp. 2948.

[86] [WG03] Z. Wang and G.B. Giannakis, "ComplexField Coding for OFDM over Fading Wireless Channels", IEEE Trans. Information Theory, Vol. 49, No. 3, March 2003, pp. 707-720.

[87] [WMG04] Z. Wang, X. Ma and G.B. Giannakis, "OFDM or Single-Carrier Block Transmissions?", IEEE Trans. Communications, Vol. 52, No. 3, March 2004, pp. 380-394.

[88] [WMS+02] H. Witschnig, T. Mayer, A. Springer, L. Maurer, M. Huemer, R. Weigel, "The Advantages of a Known Sequence versus Cyclic Prefix in a SC/FDE System", Proc. $5^{\text {th }}$ Symp. Wireless Personal Multimedia Communications WPMCO2, Oct. 2002, Honolulu, Vol. 3, pp. 1328-1332.

[89] [WWN03] D. A. Wiegandt, Z. Wu and C.R. Nassar, "High Throughput, High Performance OFDM via Pseudo-Orthogonal Carrier Interferometry Spreading Codes", IEEE Trans. Commun., Vol. 51, No. 7, July 2003, pp. 1123-1134.

[90] [XZG03] P. Xia, S. Zhou and G.B. Giannakis, "Bandwidth- and Power-Efficient Multi-Carrier Multiple Access", IEEE Trans. Commun., Nov. 2003, pp. 1828-1837.

[91] [YLF93] N. Yee, J.-P. Linnartz, and G. Fettweis, “ Multi-carrier CDMA in indoor wireless radio networks," Proc. PIMRC 1993, Yokohama, Sept. 1993, pp. 109-113.

[92] [ZK89] N. Zervos and I. Kalet, "Optimized Decision Feedback Equalizer Versus Optimized Orthogonal Frequency Division Multiplexing for High Speed Data 
Transmission over the Local Cable Network", Proc. Int. Conf. On Commun., June, 1989.

[93] [ZKT04] P. Zhu, A. Khandani and W. Tong, "Scrambling-based peak-to-average power reduction without side information", Proc. WWRF8bis, Beijing, Feb., 2004. 